\title{
Salivary Gland Carcinoma
}

National Cancer Institute

\section{Source}

National Cancer Institute. Salivary Gland Carcinoma. NCI Thesaurus. Code C9272.

A carcinoma that arises from the major or minor salivary glands. Representative examples include carcinoma ex pleomorphic adenoma, adenocarcinoma, adenoid cystic carcinoma, and mucoepidermoid carcinoma. 